American Journal of Applied Sciences 2 (9): 1279-1282, 2005

ISSN 1546-9239

(C) 2005 Science Publications

\title{
Asymptotic Hyperstability of Dynamic Systems with Point Delays
}

\author{
M. De la Sen \\ Instituto de Investigacion y Desarrollo de Procesos, Facultad de Ciencias \\ Universidad del Pais Vasco, Leioa (Bizkaia), Aptdo, 644 de Bilbao, 48080-Bilbao, Spain
}

\begin{abstract}
It is proved that a linear time-invariant system with internal point delays is asymptotically hyperstable independent of the delays if an associate delay-free system is asymptotically hyperstable and the delayed dynamics are sufficiently small.
\end{abstract}

Key words: Hyperstability, positive realness, stability independent of the delays, time-delay systems

\section{INTRODUCTION}

Global Lyapunov's stability (asymptotic stability) configurations consisting of linear time-invariant systems in the forward loop with arbitrary nonlinear (and, perhaps time-varying) devices satisfying Popov'stype time-integral inequalities are the so-called hyperstability (asymptotic hyperstability) property ${ }^{[1-3]}$. The importance of the topic relies on the fact that the stability property holds for all nonlinearity satisfying Popov's in equality for all time. In this brief, related results are obtained when the linear plant is subject to a finite number of bounded incommensurate delays (i.e. The delays are not necessarily an integer multiple of a real number) if its associated dynamics are sufficiently small. The importance of such systems is well- known in many physical systems including problems such as war /peace or population growth models, large scale systems as well as in many engineering applications as, for instance, those involving transmission and telecommunication problems ${ }^{[4-8]}$. The study of stability/hyperstability properties for systems involving external (i.e. In the inputs or outputs) delays may be addressed by direct extensions from the analysis methods concerning delay-free systems by simply transforming the relevant signals in new ones influenced by delays ${ }^{[2,4,5]}$. However, the related problems become much more involved in the case of internal (i.e. In the state) delays since the relevant dynamics possess infinitely many modes. Different techniques have been used to deal with the stability of such systems including Lyapunov's theory and its extensions as well as frequency analysis methods ${ }^{[4-8]}$. In this manuscript, the asymptotic hyperstability of continuous time-delay systems is focused on for systems including any finite number of incommensurate internal point delays of arbitrary sizes provided that the plant free of delayed dynamics satisfies a strict positive realness condition.
The obtained results are independent of the sizes of the delays provided that the delayed dynamics are sufficiently small what is evaluated in terms of sufficient smallness of the absolute value of a normalizing scalar parameter. Such results are also independent of the delayed dynamics being structured or not. The above issues are the main novelties related to previous results. The manuscript organization follows in brief the following guidelines. First, a model for the linear plant is fixed with a free parameter to then deal with the amount of tolerated delayed dynamics being compatible with the asymptotic hyperstability. Also the class of admissible feedback nonlinear/ time-varying devices satisfying a Popov's type integral inequality for all time is defined. Subsequently, a preliminary result Lemma 1 is given concerning with the sufficiency-type conditions guaranteeing strict positive realness of the transfer function for all sizes of delays and an upperbound of the absolute value of the above mentioned parameter. Then, the main asymptotic hyperstability result Theorem 1 is derived based on the intuitive physical idea that, if the transfer function is strictly positive real, then the input/output energy is strictly positive and bounded for all time what implies the asymptotic vanishing of the relevant signals in the closed loop system.

\section{FORMULATION}

Consider the open-loop linear and time-invariant single-input single-output system with an arbitrary number $\mathrm{r}$ of incommensurate internal point delays $\mathrm{h}_{\mathrm{i}}>0(\mathrm{i}=\overline{1, \mathrm{r}})$ given by:

$$
\begin{aligned}
& \dot{\mathrm{x}}(\mathrm{t})=\mathrm{Ax}(\mathrm{t})+\delta \sum_{\mathrm{i}=1}^{\mathrm{r}} \mathrm{A}_{\mathrm{i}} \mathrm{x}\left(\mathrm{t}-\mathrm{h}_{\mathrm{i}}\right)+\mathrm{bu}(\mathrm{t}) \\
& \mathrm{y}(\mathrm{t})=\mathrm{c}^{\mathrm{T}} \mathrm{x}(\mathrm{t})+\mathrm{du}(\mathrm{t})
\end{aligned}
$$

Corresponding Author: M. De la Sen, Instituto de Investigacion y Desarrollo de Procesos, Facultad de Ciencias, Universidad del Pais Vasco, Leioa (Bizkaia), Aptdo, 644 de Bilbao, 48080-Bilbao, Spain 
where, $\mathrm{x}(\mathrm{t}) \in \mathrm{R}^{\mathrm{n}}, \mathrm{u}(\mathrm{t})$ and $\mathrm{y}(\mathrm{t})$ are, respectively, the $\mathrm{n}$ state vector and the scalar input and output and $\mathrm{A}$, $\mathrm{A}_{\mathrm{i}}(\mathrm{i}=\overline{1, \mathrm{r}}), \mathrm{b} \in \mathrm{R}^{\mathrm{n}}, \mathrm{c} \in \mathrm{R}^{\mathrm{n}}$ and $\mathrm{d} \in \mathrm{R} ; \boldsymbol{\delta}$ is a scalar parameter which allows the characterization of the size of the delayed dynamics for given $\mathrm{A}_{(.)}$- matrices. The initial condition of (1) is any piecewise continuous function:

$$
\begin{aligned}
& \phi \in[-\overline{\mathrm{h}}, 0] \rightarrow \mathrm{R}^{\mathrm{n}}\left(\overline{\mathrm{h}}=\underset{1 \leq \mathrm{i} \leq \mathrm{r}}{\operatorname{Max}}\left(\mathrm{h}_{\mathrm{i}}\right)\right) . \quad \text { Assume that } \\
& \mathrm{u}(\mathrm{t})=-\Phi(\mathrm{y}(\mathrm{t})) \text { subject to: }
\end{aligned}
$$$$
\int_{0}^{\mathrm{t}} \Phi(\mathrm{y}(\tau)) \mathrm{y}(\tau) \mathrm{d} \tau \geq-\gamma_{0}^{2}
$$

for all $\mathrm{t} \geq 0$ where the constant $\gamma_{0} \neq 0$ is independent of $\mathrm{t}$ and $\Phi$ is a piecewise continuous function of time which is locally continuous within a small open neighborhood of zero with $\Phi=0$ iff $y=0$. The transfer function of (1) is:

$$
\mathrm{G}(\mathrm{s}, \delta)=\mathrm{c}^{\mathrm{T}}\left(\mathrm{sI}-\mathrm{A}-\delta \sum_{\mathrm{i}=}^{\mathrm{r}} \mathrm{A}_{\mathrm{i}} \mathrm{e}^{-\mathrm{h}_{\mathrm{i}} \mathrm{s}}\right)-1 \mathrm{~b}+\mathrm{d}
$$

where, Z (s) denotes Laplace's transform of z (t) with if $\mathrm{G}(\mathrm{s})$ is biproper and $\mathrm{d}=0$ if $\mathrm{G}(\mathrm{s})$ is strictly proper. The asymptotic hyperstability of (1) is investigated assuming that the delay-free system $\dot{z}(\mathrm{t})=\mathrm{Az}(\mathrm{t})+\mathrm{bu}(\mathrm{t})$ satisfies a strict positiveness condition. Note, in particular, that if the proper rational transfer function of such a delay-free system, namely, $\mathrm{G}_{0}(\mathrm{~s})=\mathrm{G}(\mathrm{s}, 0)=\left(\mathrm{c}^{\mathrm{T}}(\mathrm{sI}-\mathrm{A})^{-1} \mathrm{~b}+\mathrm{d}\right)$ is strictly positive real (denoted by $\mathrm{G}_{0} \in \mathrm{SPR}$ ) then $\operatorname{Re} G_{0}(s)>0$ if $\operatorname{Re} s \geq 0$ and, furthermore, all the poles of including possible zero/ pole cancellations (if any) are strictly stable (i.e. located in $\operatorname{Re} s<0)^{[9-12]}$. Thus, in addition, $\mathrm{G}_{0} \in \mathbf{R} \mathbf{H}_{\infty}$ is endowed with the $\mathrm{H}_{\infty}$ norm $\quad\left\|G_{0}\right\|_{\infty}=\operatorname{Sup}_{\omega \in R_{0}^{+}}\left(\left|G_{0}(j \omega)\right|\right) \quad$ with $\mathrm{R}_{0}^{+}=\mathrm{R}^{+} \bigcup\{0\}=\{\mathrm{s} \in \mathrm{R}: \operatorname{Re} \mathrm{s} \geq 0\}$. It is well-known that this delay- free system is asymptotically hyperstable under the same output equation as in (1) and any feedback controller (2) if $G_{0} \in S P R$. The subsequent result addresses the problem of guaranteeing sufficiently small. This fact will then allow proving the asymptotic hyperstability of (1) for any feedback $(2)^{[3,11]}$. For establishing the result, it requires the related extended definition of the squared $\mathrm{H}_{\infty}$-norm of a strictly transfer matrix $G$ according via its singular values as $\|\mathbf{G}\|_{\infty}^{2}=\operatorname{Sup}_{\omega \in \mathrm{R}_{0}^{+}}\left(\lambda_{\max }\left(\mathbf{G}^{\mathbf{T}}(\mathrm{j} \omega) \mathbf{G}(\mathrm{j} \omega)\right)\right)$. It also requires the definition of the (spectral) $1_{2}$-norm of a real matrix (or vector) $\mathrm{B}$ as $\|\mathrm{B}\|_{2}=\lambda_{\max }^{1 / 2}\left(\mathrm{~B}^{\mathrm{T}} \mathrm{B}\right)$.

Lemma 1: Assume that $d>0$ and that there exists real $\mathrm{P}=\mathrm{P}^{\mathrm{T}}>0\left(\in \mathrm{R}^{\mathrm{n} \times \mathrm{n}}\right), \mathrm{L}=\mathrm{L}^{\mathrm{T}}>0\left(\in \mathrm{R}^{\mathrm{n} \times \mathrm{n}}\right), \mathrm{q} \in \mathrm{R}^{\mathrm{n}}, \varepsilon \in \mathrm{R}^{+}$ which satisfy:

$\mathrm{A}^{\mathrm{T}} \mathrm{P}+\mathrm{PA}=-\mathrm{qq}^{\mathrm{T}}-\varepsilon \mathrm{L}$ (Lyapunov's matrix equation):

$\mathrm{Pb}-\mathrm{c}=\sqrt{\mathrm{d}} \mathrm{q}$

Assume also that:

$|\delta|<\delta_{0}=\frac{\mathrm{d}}{\alpha\left[\mathrm{d}+2 \alpha_{0}\|\mathrm{~b}\|_{2}\left(\begin{array}{c}\lambda_{\text {max }}(\mathrm{P})+ \\ \alpha_{0} \lambda_{\text {min }}(\mathrm{P})\end{array}\right)\|\mathrm{b}\|_{2}+\|\mathrm{c}\|_{2}\right]}$

where, $\alpha_{0}=\left\|(\mathrm{s} \mathrm{I}-\mathrm{A})^{-1}\right\|_{\infty}$ and:

$\alpha=\left\|(\mathrm{s} \mathrm{I}-\mathrm{A})^{-1}\left(\sum_{\mathrm{i}=}^{\mathrm{r}} \mathrm{A}_{\mathrm{i}} \mathrm{e}^{-\mathrm{h}_{\mathrm{i}} \mathrm{s}}\right)\right\|_{\infty} \leq \alpha_{0} \beta$

With:

$\beta=\sum_{i=1}^{r}\left\|A_{i}\right\|_{2}$

Thus, $\mathrm{G} \in \mathrm{SPR}$ for all real $\delta$ satisfying $|\delta| \in\left[0, \delta_{0}\right)$ [in particular, $\mathrm{G}_{0} \in \mathrm{SPR}$ ].

Remarks: (1) Lemma 1 guarantees that A is a stability matrix through Lyapunov's equation in (4). This is necessary for $\mathrm{G}_{0} \in \mathrm{SPR}$ what implies also that $\mathrm{G} \in \mathrm{RH}_{\infty}$ both being biproper since $\mathrm{d} \neq 0$. Thus, all zero/pole cancellations in $\mathrm{G}_{0}(\mathrm{~s})$, if any, are strictly stable what is always guaranteed if $\left(\mathrm{c}^{\mathrm{T}}, \mathrm{A}\right)$ is detectable and $(\mathrm{A}, \mathrm{b})$ is stabilizable. Note that if $\mathrm{d}=0$ then $G_{0}(s)$ and $G(s)$ are strictly proper and then at most (non strictly) positive real.

Lemma 1 may be used with $\mathrm{q}=0$ implying $\mathrm{c}=\mathrm{P}$ b.

Proof: Define $\quad \Delta(\mathrm{j} \omega)=\sum_{\mathrm{i}=1}^{\mathrm{r}} \mathrm{A}_{\mathrm{i}} \mathrm{e}^{-\mathrm{j} \omega \mathrm{h}_{\mathrm{i}}} \quad$ and $m^{\mathrm{T}}(j \omega)=\mathrm{c}^{\mathrm{T}} \mathrm{m}_{0}^{\mathrm{T}}(j \omega)$ with

$\mathrm{m}_{0}^{\mathrm{T}}(\mathrm{j} \omega)=\left(\mathrm{I}-\delta(\mathrm{j} \omega \mathrm{I}-\mathrm{A})^{-1} \Delta(\mathrm{j} \omega)\right)^{-1}$.

Which exists for all real $\omega$ if $1>|\delta| \operatorname{Sup}_{\omega \in \mathrm{R}_{0}^{+}}\left(\left|(j \omega I-A)^{-1} \Delta(j \omega)\right|\right)$ 
$\mathrm{m}_{0}^{\mathrm{T}}(j \omega)=\left(\mathrm{I}-\delta(j \omega \mathrm{I}-\mathrm{A})^{-1} \Delta(\mathrm{j} \omega)\right)^{-1}$ which exists for all real $\omega$ if $1>|\delta| \operatorname{Sup}_{\omega \in R_{0}^{+}}\left(\left|(j \omega I-A)^{-1} \Delta(j \omega)\right|\right)$.

What is, in particular, ensured if (5) holds). Note that $\mathrm{m}_{0}(j \omega)=\mathrm{I}+\mathrm{P}^{\prime}(j \omega)$ with

$P^{\prime}(j \omega)$

$=\delta(j \omega I-A)^{-1} \Delta(j \omega)\left(I-\delta(j \omega I-A)^{-1} \Delta(j \omega)\right)^{-1}$

Such that:

$\left\|\mathrm{P}^{\prime}\right\|_{\infty} \leq \frac{|\delta| \alpha}{1-|\delta| \alpha}$ if $|\delta|<\alpha^{-1}$

Thus, direct calculations using (6)-(7) as well as the odd symmetry of the involved frequency response curves lead to:

$$
\begin{aligned}
& 2 \operatorname{Re} G(j \omega)=G(j \omega)+G^{T}(-j \omega)= \\
& 2 d+m^{T}(j \omega)(j \omega I-A)^{-1} b-b^{T}\left(j \omega I+A^{T}\right)^{-1} m(-j \omega) \\
& \geq d+\left(q^{T}(j \omega I-A)^{-1} b-\sqrt{d}\right)^{2}- \\
& \varepsilon b^{T}\left(j \omega I+A^{T}\right)^{-1} L(j \omega I-A)^{-1} b+ \\
& \left\{b^{T}\left(-j \omega I-A^{T}\right)^{-1}\left(P P^{T}(j \omega)-P^{\prime}(-j \omega) P\right)(j \omega I-A)^{-1} b\right\} \\
& +\sqrt{d}\left\{\begin{array}{c}
b^{T}\left(-j \omega I-A^{T}\right)^{-1} P^{\prime}(-j \omega) \\
q+q^{T} P^{T}(j \omega)(j \omega I-A)^{-1} b
\end{array}\right\} \\
& \geq d+\varepsilon|T(j \omega)|^{2}-2 \frac{\alpha \alpha_{0}|\delta|\|b\|_{2}}{1-|\delta| \alpha} \\
& {\left[\left(\lambda_{\max }(P)+\alpha_{0} \lambda_{\min }(P)\right)\|b\|_{2}+\|c\|_{2}\right]}
\end{aligned}
$$

where, $\quad T(j \omega)=L^{\prime T}(j \omega I-A)^{-1} b \quad$ with $L^{\prime} \quad$ such that $\mathrm{L}=\mathrm{L}^{\prime} \mathrm{L}^{\prime}>0$. The last identity of (8) is greater than zero if (5) holds and the proof follows directly.

Theorem 1 (main result of asymptotic hyperstability): Assume that $\mathrm{G} \in \mathrm{SPR}$ what is guaranteed if Lemma 1 holds. Thus, the closed-loop system (1)-(2) is asymptotically hyperstable; i.e. (1) is globally Lyapunov's stable for all feedback control law (2), for any real $\delta$ satisfying $|\delta| \in\left[0, \delta_{0}\right)$. That property holds independently of the sizes of the delays.

Proof: Define truncated time signals $\mathrm{z}_{\mathrm{t}}(\tau)=\mathrm{z}(\tau)$ for all $\tau \in[0, \mathrm{t}]$ and $\mathrm{z}_{\mathrm{t}}(\tau)=0$, otherwise), of the Fourier transform $Z_{t}(j \omega)$ for any vector signal $z(t)$, of Fourier transform $Z(j \omega)$, for any time $t \geq 0$ and any frequency $\omega$. From Parseval's theorem relating the input- output energy in the time and frequency domains, (1)-(2), $\mathrm{G} \in \mathrm{SPR}$ from Lemma 1 and $\operatorname{Im} \mathrm{G}(\mathrm{j} \omega)=-\operatorname{Im} \mathrm{G}(-\mathrm{j} \omega)$ for all $\omega$, one gets for all $\mathrm{t} \geq 0$ :

$$
\begin{aligned}
& 0<\operatorname{Inf}_{\omega \in \mathrm{R}_{0}^{+}}(\operatorname{Re} G(j \omega)) \int_{0}^{\mathrm{t}} \mathrm{u}^{2}(\tau) \mathrm{d} \tau \\
& \leq \int_{-\infty}^{\infty} \mathrm{G}(j \omega)\left|\mathrm{U}_{\mathrm{t}}(j \omega)\right|^{2} \mathrm{~d} \omega=\int_{-\infty}^{\infty} \mathrm{Y}_{\mathrm{t}}(j \omega) \mathrm{U}_{\mathrm{t}}^{2}(-j \omega)^{2} \mathrm{~d} \omega \\
& =2 \pi \int_{-\infty}^{\infty} \mathrm{y}_{\mathrm{t}}(\tau) \mathrm{u}_{\mathrm{t}}(\tau) \mathrm{d} \tau=2 \pi \int_{0}^{\mathrm{t}} \mathrm{y}(\tau) \mathrm{u}(\tau) \mathrm{d} \tau \\
& \leq 2 \pi \gamma_{0}^{2}<\infty
\end{aligned}
$$

These expressions dictate that the input/output product time-integral (a measure of the input/output energy) is monotonically non-decreasing with time since it is positive and strictly positive for all $t>0$ since $\operatorname{Re} G(j \omega)>0$ so that $u(t)$ is bounded piecewise continuous on $\mathrm{R}_{0}^{+}$and converges asymptotically to zero as $t \rightarrow \infty$ except (potentially) over a set of zero measure of bounded discontinuities. Thus, $\mathrm{y}(\mathrm{t}) \rightarrow 0$ as $\mathrm{t} \rightarrow \infty$ and is bounded on $\mathrm{R}_{0}^{+}$since $\mathrm{G} \in \mathrm{R} \mathrm{H}_{\infty}$. Now, since the nonlinear feedback function $\Phi($.$) eq. 2$, is locally continuous around the equilibrium, $y(t) \rightarrow 0$ implies $\mathrm{u}(\mathrm{t}) \rightarrow 0$ as $\mathrm{t} \rightarrow \infty$ so that the above mentioned set of potential discontinuities of $\mathrm{u}(\mathrm{t})$ is empty after some finite time. As a result, both $\mathrm{u}(\mathrm{t})$ and $\mathrm{y}(\mathrm{t})$ converge asymptotically to zero.

\section{EXAMPLE}

Consider $\quad(1) \quad$ with $\left.\quad \begin{array}{cc}\mathrm{n}=2, & \mathrm{r}=1 ; \\ 0 & 1 \\ -\mathrm{a} \beta & -(\beta+\mathrm{a})\end{array}\right]$
$\mathrm{b}^{\mathrm{T}}=(-1, \beta) \mathrm{k} ; \mathrm{c}^{\mathrm{T}}=(1,0)$ and $\mathrm{A}=\left[\begin{array}{cc}0 & 1 \\ -\mathrm{a} & -1\end{array}\right]$ with $\mathrm{a}>0, \beta>0$ and $\mathrm{d}>0$. The open-loop forward-loop is globally asymptotically stable if $|\delta|<\left|\frac{\mathrm{a}}{\mathrm{a}_{1}}\right|$ and $\mathrm{A}$ is a stability matrix. Thus (3)-(4) hold with $q=(P b-c) / \sqrt{d}$, and:

$\mathrm{L}=\varepsilon^{-1}\left(\mathrm{~L}_{1}-\mathrm{d}^{-1}\left(\mathrm{~Pb} b^{\mathrm{T}} \mathrm{P}+\mathrm{c} \mathrm{c}^{\mathrm{T}}-\mathrm{Pbc} \mathrm{c}^{\mathrm{T}}-\mathrm{cb}^{\mathrm{T}} \mathrm{P}\right)\right)$

Being symmetric (and positive definite for sufficiently small $\varepsilon>0$ ) for arbitrary $L_{1}=L_{1}^{T}>0$. A simple calculation combining those constraints yields $\mathrm{P}=\int_{0}^{\infty} \mathrm{e}^{\mathrm{A}^{\mathrm{T}} \tau} \mathrm{L}_{1} \mathrm{e}^{\mathrm{A} \tau} \mathrm{d} \tau$ then satisfying the matrix Lyapunov Equation $\mathrm{A}^{\mathrm{T}} \mathrm{P}+\mathrm{PA}=-\mathrm{L}_{1}$.

Thus, from Lemma $1, G \in \mathrm{SPR}$ if $\delta_{0}$ in (5) is calculated by using $\alpha \leq \alpha_{0}\left|\mathrm{a}_{1}\right|$ and: 
$\alpha_{0} \leq \sqrt{1+\left(\frac{(\beta+a)^{2}+(\beta+a)}{a^{2} \beta^{2}}\right)}$

Obtained from the calculations of the related $\mathrm{H}_{\infty}$ norms. Thus, from Theorem 1, the feedback configuration (1)-(2) is asymptotically hyperstable for any nonlinear device satisfying the constraint in (2). The associate transfer function possesses a strictly stable zero/pole cancellation at $s=-\beta$ which has not been taken into account in the above calculations. This is reasonable when the transfer function numerator and denominator are not factored explicitly from the statespace description especially for higher order systems. If such a cancellation is known "a priori" and removed for a minimum state-space realization of (1) resulting in:

$\mathrm{A}=-\mathrm{a}, \mathrm{A}_{1}=-\mathrm{a}, \mathrm{b}=\mathrm{k}, \mathrm{c}=1$ then

$\delta_{0}=\frac{\mathrm{ada}}{\left|\mathrm{a}_{1}\right|(\mathrm{da}+2 \mathrm{k}(\mathrm{a}+1) \mathrm{a})}$

From Lemma 1 with $\mathrm{P}=1 / \mathrm{k}, \mathrm{q}=0$. In this simple example, the calculations may also be performed from the real part of the transfer function once the cancellation, if known, is removed. In this case, this leads to $d>0, \delta_{0}=\frac{a}{\left|a_{1}\right|}$ which is the weakest found constraint. However, obtaining factored transfer functions from a state-space realization is not easy for high-order systems in the presence of delays. This fact justifies the adequacy of the proposed method to practical problems.

\section{ACKNOWLEGMENTS}

The author is grateful to MCYT and UPV /EHU for their partial support of this work.

\section{REFERENCES}

1. Popov, V.M., 1970. Hyperstability of Control Systems. Berlin, Springer-Verlag.
2. Popov, V.M. and A. Halanay, 1963. On the stability of nonlinear automatic control systems with lagging argument. Autom. Remote Control, 23: 783-786.

3. de la Sen, M., 1986. Stability of composite systems with an asymptotically hyperstable block. Intl. J. Control, 44: 1769-1775.

4. Gorecki, V., S. Fuska, P. Grabowski and A. Korytowski, 1989. Analysis and Synthesis of TimeDelay Systems. John Wiley and Sons, Varszawa.

5. Niculescu, S.I., 2001. Delay Effects on Stability. A Robust Control Approach, Lecture Notes in Control and Information. Series No. 269. M. Thoma and M. Morari (Eds.), Springer-Verlag, Berlin.

6. De la Sen, M. and Ningsu Luo, 2003. A note on the stability of linear time-delay systems with impulsive inputs. IEEE Trans. Circuits and Syst. I, 50: 149-152.

7. Liu, P.L. and T.J. Su, 1998. Robust stability of interval time-delay systems with delay dependence. Syst. Control Lett., 33: 231-239.

8. Lien, C.H., 1999. Asymptotic criterion for neutral systems with multiple time delays. Electron. Lett., 35: 850-852.

9. Marquez, H.J. and C.J. Damaren, 1995. On the design of strictly positive real transfer functions. IEEE Trans. Circuits and Sys. I, 42: 214218.

10. Gregor, J., 1996. On the design of positive real functions. IEEE Trans. Circuits and Syst. I, 43: 945-947.

11. De la Sen, M., 1999. Relationships between positive realness of continuous transfer functions and their digital counterparts. Electron. Lett., 35: 1298-1299.

12. De la Sen, M., 1998. A method for general design of positive real functions. IEEE Trans. Circuits and Sys. I, 45: 764-769. 